\title{
Circular RNA ABCB10 promotes cell proliferation and invasion, but inhibits apoptosis via regulating the microRNA-1271-mediated Capn4/Wnt// -catenin signaling pathway in epithelial ovarian cancer
}

\author{
XUEFANG LIN, YAN CHEN, XIABIN YE and XUEMEI XIA \\ Department of Gynaecology and Obstetrics, People's Hospital of Lishui City, The Sixth Affiliated Hospital of \\ Wenzhou Medical University, The Affiliated Hospital of Lishui College, Lishui, Zhejiang 323000, P.R. China
}

Received January 3, 2020; Accepted July 20, 2020

DOI: $10.3892 / \mathrm{mmr} .2021 .12026$

\begin{abstract}
Circular RNA ABCB10 (circ-ABCB10) modulates cellular functions and microRNA (miR)-1271 in epithelial ovarian cancer (EOC). The present study aimed to investigate the interaction between circ-ABCB10 and miR-1271 in regulating EOC cellular function and the calpain small subunit 1 (Capn4)/Wnt/ $\beta$-catenin signaling pathway. circ-ABCB10 and miR-1271 expression levels were detected in EOC cells (OVCAR3, UWB1.289, SKOV3 and CAOV3) and normal ovarian epithelial cells (IOSE80) via reverse-transcription quantitative PCR. SKOV3 cells were transfected with control short hairpin (sh)RNA plasmids, control inhibitor, circ-ABCB10 shRNA plasmids and miR-1271 inhibitor. UWB1.289 cells were transfected with control overexpression plasmids, control mimic, circ-ABCB10 overexpression plasmids and miR-1271 mimic. Subsequently, cell proliferation, apoptosis, invasion and the Capn $4 / \mathrm{Wnt} / \beta$-catenin signaling pathway were assessed. In addition, a luciferase activity assay was performed. circ-ABCB10 expression was significantly increased in OVCAR3, SKOV3 and CAOV3 cells compared with IOSE80 cells, but was not significantly altered in UWB1.289 cells. miR-1271 expression was significantly decreased in OVCAR3, UWB1.289, SKOV3 and CAOV3 cells compared with IOSE80 cells. In both SKOV3
\end{abstract}

Correspondence to: Dr Xuemei Xia, Department of Gynaecology and Obstetrics, People's Hospital of Lishui City, The Sixth Affiliated Hospital of Wenzhou Medical University, The Affiliated Hospital of Lishui College, 15 Dazhong Street, Liandu, Lishui, Zhejiang 323000, P.R. China

E-mail: 080884821@163.com

Abbreviations: EOC, epithelial ovarian cancer; circ-ABCB10, circular RNA ABCB10; circRNA, circular RNA; FOXR2, forkhead box 2

Key words: epithelial ovarian cancer, circular RNA ATP binding cassette subfamily B member 10 , calpain small subunit $1 / \mathrm{Wnt} / \beta$-catenin, cell proliferation, cell apoptosis, cell invasion and UWB1.289 cells, circ-ABCB10 negatively regulated miR-1271, whereas miR-1271 did not affect circ-ABCB10. Furthermore, circ-ABCB10 enhanced cell proliferation, invasion and the Capn $4 / \mathrm{Wnt} / \beta$-catenin signaling pathway, but inhibited cell apoptosis, whereas miR-1271 suppressed cell proliferation, invasion and the Capn $4 / \mathrm{Wnt} / \beta$-catenin signaling pathway, but facilitated cell apoptosis. Moreover, miR-1271 attenuated the proproliferative, proinvasive and antiapoptotic effects of circ-ABCB10, and reversed the positive regulation of circ-ABCB10 on the Capn $4 / \mathrm{Wnt} / \beta$-catenin signaling pathway. Besides, the luciferase activity assay indicated that circ-ABCB10 directly bound to miR-1271. In conclusion, the present study indicated that circ-ABCB10 promoted cell proliferation and invasion, and suppressed apoptosis by regulating the miR-1271-mediated Capn $4 / \mathrm{Wnt} / \beta$-catenin signaling pathway in EOC.

\section{Introduction}

Epithelial ovarian cancer (EOC) accounts for $~ 90 \%$ of ovarian cancer cases, which is the most common cause of gynaecological cancer-related death, causing $>150,000$ deaths annually worldwide $(1,2)$. Due to the asymptomatic nature of EOC at early stages and the lack of an effective screening program for assessing disease risk, patients are generally diagnosed at an advanced stage (International Federation of Gynecology and Obstetrics stage III and IV), which is associated with the dissemination of cancer cells from the ovary to the peritoneal cavity $(1,3,4)$. Surgical resection and platinum-based chemotherapy are the standard treatment strategies for patients with EOC (4). However, the majority of patients develop chemoresistance and recurrence, which results in a poor 5-year relative survival rate (5). To improve the prognosis in patients with EOC, identifying the molecular pathogenies underlying EOC is important for the development of novel and effective drug targets.

Circular RNA (circ/circRNA)-ABCB10, a novel circRNA, exerts oncological effect in several types of cancer, such as breast and non-small-cell lung cancer (6-8). As for EOC, our previous study revealed that circ-ABCB10 correlates with deteriorated clinical features and poor prognosis in 
patients with EOC. Moreover, the previous study indicated that circ-ABCB10 facilitates cell proliferation, inhibits cell apoptosis and negatively regulates microRNA (miR)-1271, miR-1252 and miR-203 in EOC cells (9). Meanwhile, miR-1271 suppresses cell proliferation and invasion by downregulating calpain small subunit 1 (Capn4), and Capn4 enhances EOC cell proliferation and migration by targeting the $\mathrm{Wnt} / \beta$-catenin signaling pathway $(10,11)$. Based on the aforementioned studies, it was hypothesized that circ-ABCB10 might competitively interact with miR-1271 to promote EOC progression by activating Capn 4 and its downstream Wnt/ $\beta$-catenin signaling pathway. Therefore, the present study aimed to investigate the interaction between circ-ABCB10 and miR-1271 in regulating cell proliferation, apoptosis, invasion and the Capn $4 / \mathrm{Wnt} / \beta$-catenin signaling pathway in EOC.

\section{Materials and methods}

Cell culture. Human EOC cell lines (OVCAR3, UWB1.289, SKOV3 and CAOV3) were purchased from American Type Culture Collection and a human normal ovarian epithelial cell line (IOSE80) was purchased from BioVector NTCC, Inc. Cells were cultured as previously described (9). OVCAR3, UWB1.289, SKOV3 and IOSE80 cell lines were cultured in 90\% RPMI-1640 medium (Gibco; Thermo Fisher Scientific,Inc.) and 10\% FBS (Gibco; Thermo Fisher Scientific, Inc.); CAOV3 cell line was cultured in 90\% DMEM (Gibco; Thermo Fisher Scientific, Inc.) and 10\% FBS (Gibco; Thermo Fisher Scientific, Inc.); all cell lines were cultured under $95 \%$ air and $5 \% \mathrm{CO}_{2}$ at $37^{\circ} \mathrm{C}$. Following culture, the relative expression levels of circ-ABCB10 and miR-1271 in cells were determined via reverse transcription-quantitative PCR (RT-qPCR).

Cell transfection. Control overexpression plasmids (100 nM), circ-ABCB10 overexpression plasmids (100 nM), control shRNA plasmids (100 nM) and circ-ABCB10 shRNA plasmids (100 nM) were constructed by Shanghai GenePharma Co., Ltd. using pEX-2 and pGPU6. Control mimic (100 nM; 5'-AAC ACCGAACGAGACAGGATT-3'), miR-1271 mimic (100 nM; 5'-CUUGGCACCUAGCAAGCACUCA-3'), control inhibitor (100 nM) (5'-AAGAACAACACAAAAGAACAG-3') and miR-1271 inhibitor (100 nM; 5'-UGAGUGCUUGCUAGGUGC CAAG-3') were also synthesized by Shanghai GenePharma Co., Ltd. SKOV3 $\left(5 \times 10^{5}\right.$ cells/well) and UWB1.289 cells $\left(5 \times 10^{5}\right.$ cells/well) were transfected using HilyMax (Dojindo Molecular Technologies, Inc.). The following four groups were generated using SKOV3 cells: i) NC (-), cells co-transfected with control shRNA plasmid and control inhibitor; ii) circ (-), cells co-transfected with circ-ABCB10 shRNA plasmid and control inhibitor; iii) miR (-), cells co-transfected with miR-1271 inhibitor and control shRNA plasmid; and iv) circ (-) + miR (-), cells co-transfected with circ-ABCB10 shRNA plasmid and miR-1271 inhibitor. The following four groups were generated using UWB1.289 cells: i) NC(+), cells co-transfected with control overexpression plasmid and control mimic; ii) circ $(+)$, cells co-transfected with circ-ABCB10 overexpression plasmid and control mimic; iii) $\mathrm{miR}(+)$, cells co-transfected with miR-1271 mimic and control overexpression plasmid; and iv) circ $(+)$ + miR (+), cells co-transfected with circ-ABCB10 overexpression plasmid and miR-1271 mimic. At $24 \mathrm{~h}$ post-transfection, the relative expression levels of circ-ABCB10 and miR-1271 were detected via RT-qPCR.

Cell proliferation, apoptosis and invasion. Cell proliferation was assessed at $0,24,48$ and $72 \mathrm{~h}$ post-transfection using a Cell Counting Kit-8 assay (Sigma-Aldrich; Merck KGaA) according to the manufacturer's protocol. At $48 \mathrm{~h}$ post-transfection, cell apoptosis was assessed using an Annexin V-FITC Apoptosis Detection kit (Sigma-Aldrich; Merck KGaA), according to the manufacturer's protocol. Apoptotic cells (early and late apoptosis) were detected via flow cytometry using CytoFLEX system (Beckman Coulter, Inc.), and FlowJo software 7.6 (FlowJo LLC) was used for data analysis. In addition, at $48 \mathrm{~h}$ post-transfection, cell invasion was evaluated by performing a Transwell assay. Each Transwell insert (size, $8 \mu \mathrm{m}$; Corning, Inc.) was pre-coated

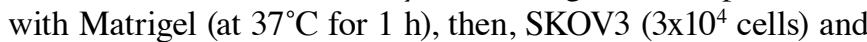
UWB1.289 cells ( $3 \times 10^{4}$ cells) suspended in serum-free culture medium (Gibco; Thermo Fisher Scientific, Inc.) were added into the upper chamber (Corning). Culture medium containing 10\% FBS (Gibco; Thermo Fisher Scientific, Inc.) was added into the lower chamber. After $24 \mathrm{~h}$ of incubation, cells that invaded the Matrigel coated filters were fixed with methanol (Sigma-Aldrich; Merck KGaA), stained with crystal violet (at $37^{\circ} \mathrm{C}$ for $15 \mathrm{~min}$; Sigma-Aldrich; Merck KGaA) and counted with a BX 41 inverted microscope under x200 magnification (Olympus Corporation).

$R T-q P C R$. Total RNA was isolated from IOSE80, OVCAR3, UWB1.289, SKOV3 and CAOV3 cells using TRIzol ${ }^{\circledR}$ reagent (Invitrogen; Thermo Fisher Scientific, Inc.). Linear RNA was digested using RNase R enzyme (Epicentre; Illumina, Inc.) for the detection of circ-ABCB10, but linear RNA digestion was not conducted for the detection of miR-1271, Capn4, Wnt1, $\beta$-catenin, GAPDH and U6. Total RNA was reverse transcribed into cDNA using the PrimeScript RT reagent kit (Takara Bio, Inc.). The synthetization of cDNA was performed at $37^{\circ} \mathrm{C}$ for $15 \mathrm{~min}$ and $85^{\circ} \mathrm{C}$ for $5 \mathrm{sec}$. Subsequently, qPCR was performed using the QuantiNova SYBR Green PCR kit (Qiagen $\mathrm{GmbH}$ ) at $95^{\circ} \mathrm{C}$ for $30 \mathrm{sec}$, followed by 40 cycles of amplification $\left(95^{\circ} \mathrm{C}\right.$ for $5 \mathrm{sec}$ and $60^{\circ} \mathrm{C}$ for $\left.19 \mathrm{sec}\right)$. The following primers were used for qPCR: circ-ABCB10 forward, 5'-GTGCTGATGACCCTTCCT CTG-3' and reverse, 5'-GGGAATCCTGAGTGACTTTGG T-3'; GAPDH forward, 5'-GAGTCCACTGGCGTCTTCAC-3' and reverse, 5'-ATCTTGAGGCTGTTGTCATACTTCT-3'; miR-1271 forward, 5'-ACACTCCAGCTGGGCTTGGCACCT AGCAAG-3' and reverse, 5'-TGTCGTGGAGTCGGCAAT TC-3'; U6 forward, 5'-CGCTTCGGCAGCACATATACTA-3' and reverse, 5'-ATGGAACGCTTCACGAATTTGC-3'; Capn4 forward, 5'-ACCCACTCCGTAACCTC-3' and reverse, 5'-GGG TAGCAACCGTGAA-3'; Wnt1 forward, 5'-TACCTCCAGTCA CACTCCCC-3' and reverse, 5'-CCATGGCAGGAGAATAGG AA-3'; and $\beta$-catenin forward, 5'-GCCATTACAACTCTCCAC AACCT-3' and reverse, 5'-GACAGATAGCACCTTCAGCAC TC-3'. circRNA and mRNA expression levels were normalized to the internal reference gene GAPDH. miRNA expression levels were normalized to the internal reference gene U6. Expression levels were quantified using the $2^{-\Delta \Delta \mathrm{Cq}}$ method (12).

Western blotting. Western blotting was performed as previously described (9). The following antibodies were used: 
A

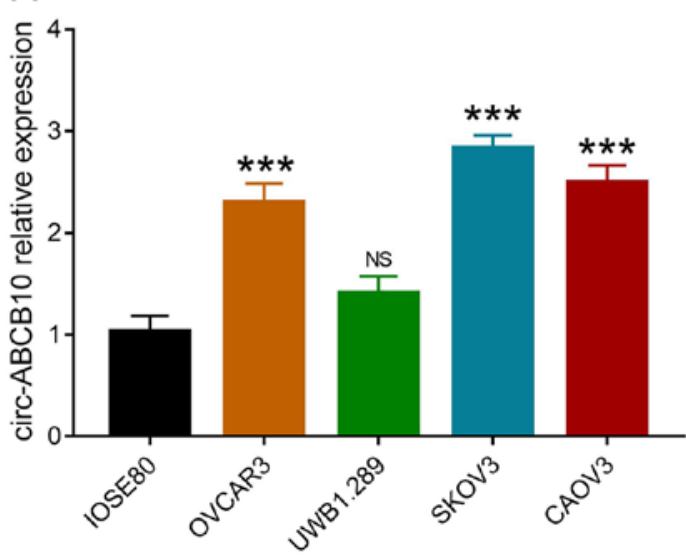

B

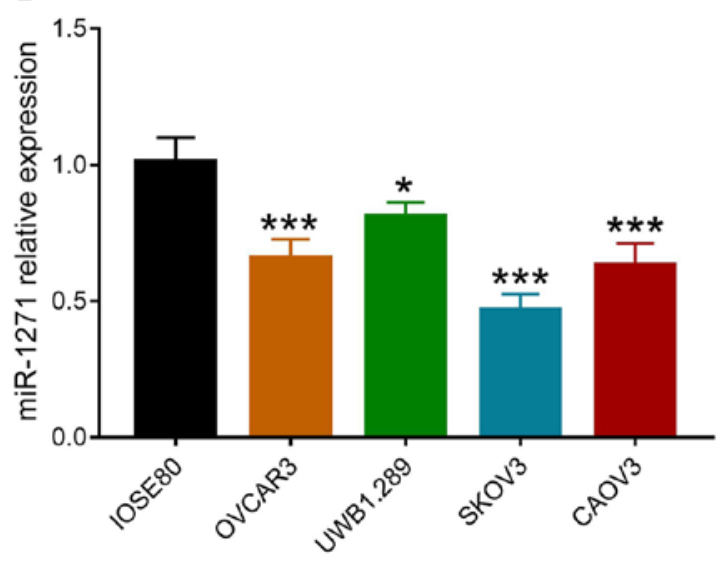

Figure 1. circ-ABCB10 and miR-1271 relative expression levels in human EOC cells and human normal ovarian epithelial cells. (A) circ-ABCB10 and (B) miR-1271 expression levels in human EOC cells (OVCAR3, UWB1.289, SKOV3 and CAOV3) and human normal ovarian epithelial cells (IOSE80). ${ }^{*} \mathrm{P}<0.05,{ }^{* * *} \mathrm{P}<0.001$; NS, not significant $(\mathrm{P}>0.05)$. circ, circular RNA ABCB10; miR-1271, microRNA-1271; EOC, epithelial ovarian cancer; NS, not significant.

Capn4 (1:1,000; cat. no. ab92356; Abcam), Wnt1 (1:500; cat. no. ab15251; Abcam), $\beta$-catenin (1:400; cat. no. ab68183; Abcam), GAPDH (1:10,000; cat. no. ab128915; Abcam) and HRP-conjugated goat anti-rabbit IgG H\&L (1:10,000; cat. no. ab6721; Abcam).

Luciferase activity assay. The wild-type (WT) and mutant (MUT) circ-ABCB10 recombinant plasmids were constructed using the psiCHECK luciferase vector (Hanbio Biotechnology Co., Ltd.). 293T cells (5x10 5 cells/well) (American Type Culture Collection) were co-transfected with WT-circ-ABCB10 $(100 \mathrm{nM})$ or MUT-circ-ABCB10 (100 nM) and miR-1271 mimic or control mimic (100 nM) using HilyMax (Dojindo Molecular Technologies, Inc.). The following four groups were generated: i) $\mathrm{WT}$ + miR-1271; ii) WT + control; iii) MUT + miR-1271; and iv) MUT + control. Luciferase activity was measured using the luciferase activity detecting kit (Biothrive) with the Dual-Luciferase Reporter Assay system (Promega Corporation) $48 \mathrm{~h}$ after transfection, and the Firefly luciferase activity was normalized to the Renilla luciferase activity.

Statistical analysis. Data are presented as the mean \pm standard deviation of three experimental repeats. Comparisons between two groups were analyzed using the unpaired Student's t-test. Comparisons among multiple groups were analyzed using one-way ANOVA followed by Dunnett's or Tukey's post hoc test. $\mathrm{P}<0.05$ was considered to indicate a statistically significant difference. Statistical analyses were performed by SPSS 22.0 Software (IBM Corp.), and graphs were plotted by GraphPad Prism 7.02 Software (GraphPad Software, Inc.).

\section{Results}

Comparison of circ-ABCB10 and miR-1271 expression levels between EOC and normal ovarian epithelial cells. Compared with IOSE80 cells, circ-ABCB10 expression was significantly increased in OVCAR3, SKOV3 and CAOV3 cells, but was not significantly altered in UWB1.289 cells (Fig. 1A). miR-1271 expression was significantly decreased in OVCAR3, UWB1.289, SKOV3 and CAOV3 cells compared with IOSE80 cells (Fig. 1B). Therefore, SKOV3 and UWB1.289 cells were selected for subsequent experiments.

circ-ABCB10 and miR-1271 expression levels following transfection. In SKOV3 cells, circ-ABCB10 expression levels were significantly decreased in the circ (-) group compared with the NC (-) group, but were similar between the miR (-) group and the NC (-) group, as well as between the circ (-) + miR (-) and circ (-) groups (Fig. 2A). miR-1271 expression levels were significantly decreased in the miR (-) group compared with the NC (-) group, and in the circ (-) + miR (-) group compared with the circ (-) group. By contrast, miR-1271 expression levels were significantly increased in the circ (-) group compared with the NC (-) group (Fig. 2B). In UWB1.289 cells, circ-ABCB10 expression levels were significantly increased in the circ (+) group compared with $\mathrm{NC}(+)$ group, but similar between the miR $(+)$ group and the $\mathrm{NC}(+)$ group, as well as between the circ $(+)+$ miR $(+)$ group and the circ (+) group (Fig. 2C). Furthermore, miR-1271 relative expression levels were significantly elevated in the $\mathrm{miR}(+)$ group compared with the $\mathrm{NC}(+)$ group, and in the circ $(+)+$ miR $(+)$ group compared with the circ $(+)$ group. By contrast, miR-1271 expression was significantly decreased in the circ $(+)$ group compared with the NC (+) group (Fig. 2D). Collectively, the results indicated that circ-ABCB10 negatively regulated miR-1271, whereas miR-1271 did not regulate circ-ABCB10 in EOC.

circ-ABCB10 promotes cell proliferation by targeting $m i R-1271$. In SKOV3 cells, cell proliferation was significantly increased in the miR (-) group compared with NC (-) group at 48 and $72 \mathrm{~h}$. Similarly, cell proliferation was significantly increased in the circ (-) + miR (-) group compared with circ (-) group at 48 and $72 \mathrm{~h}$, but significantly decreased in the circ (-) group compared with the NC (-) group at 48 and $72 \mathrm{~h}$ (Fig. 3A).In UWB1.289 cells, cell proliferation was significantly reduced in the miR $(+)$ group compared with the $\mathrm{NC}(+)$ group at $72 \mathrm{~h}$, and in the circ $(+)+\mathrm{miR}(+)$ group compared with the circ (+) group at 48 and $72 \mathrm{~h}$. By contrast, cell proliferation was significantly enhanced in the circ $(+)$ group compared with the $\mathrm{NC}(+)$ group at 48 and $72 \mathrm{~h}$ (Fig. 3B). Collectively, the results 


\section{SKOV3 cells}

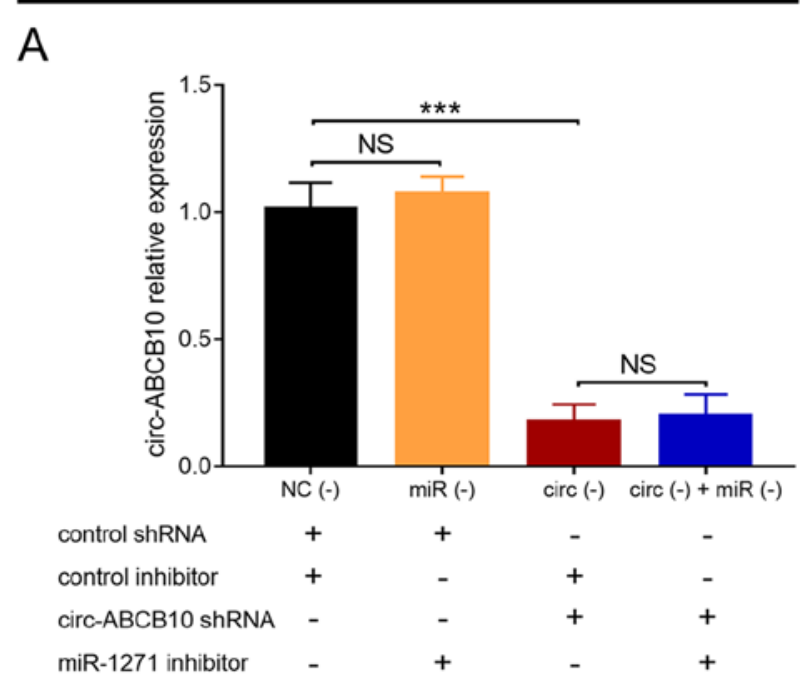

B

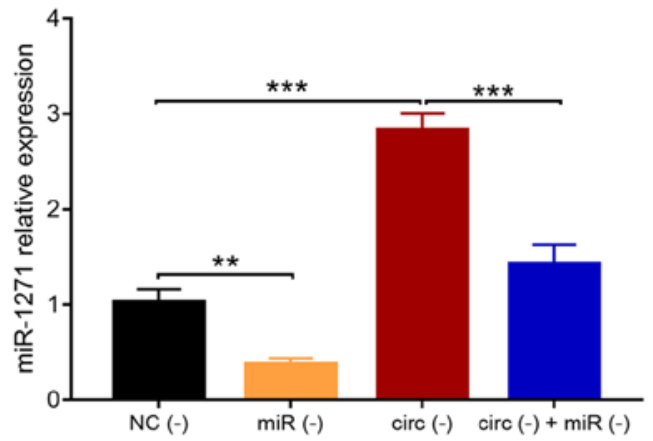

control shRNA

control inhibitor

circ-ABCB10 shRNA

miR-1271 inhibitor
$+$
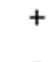

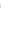

$+$

$+$

$+$

-

Figure 2. Interaction between circ-ABCB10 and miR-1271. (A) circ-ABCB10 and (B) miR-1271 expression levels in NC (-), miR (-), circ (-) and circ (-) + miR (-) SKOV3 cells. (C) circ-ABCB10 and (D) miR-1271 expression levels in NC (+), miR (+), circ (+) and circ (+) + miR (+) UWB1.289 cells. ${ }^{* *} \mathrm{P}<0.01,{ }^{* * *} \mathrm{P}<0.001$. circ-ABCB10, circular RNA ABCB10; miR-1271, microRNA 1271; NC, negative control; NS, not significant; shRNA, short hairpin RNA; OR, overexpression.

SKOV3 cells

A

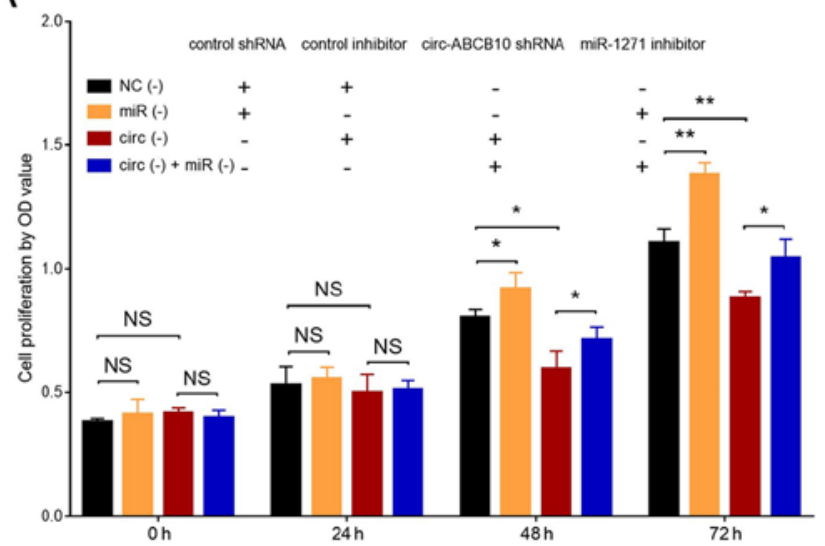

UWB1.289 cells

$\mathrm{B}$

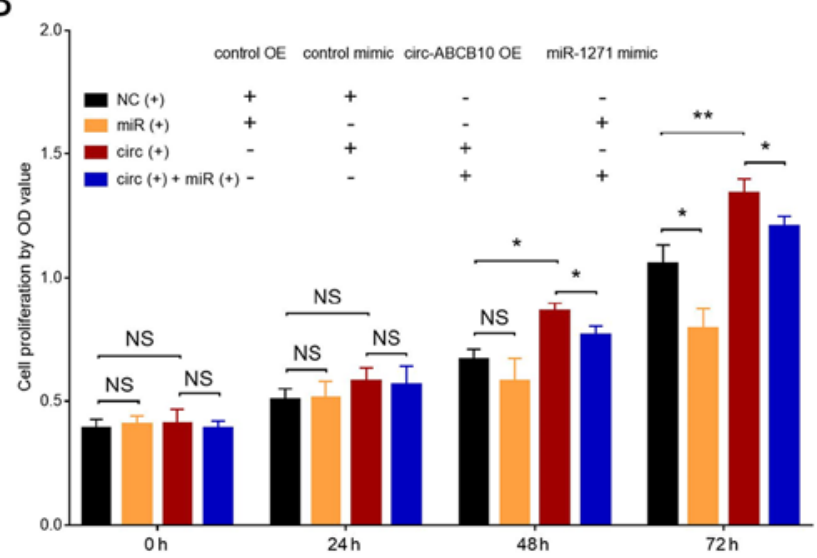

Figure 3. Effect of circ-ABCB10 and miR-1271 on cell proliferation. Cell proliferation at $0,24,48$ and $72 \mathrm{~h}$ in (A) NC (-), miR (-), circ (-) and circ (-) + miR (-) SKOV3 cells, and (B) NC (+), miR (+), circ (+) and circ (+) + miR (+) UWB1.289 cells. ${ }^{*} \mathrm{P}<0.05,{ }^{* *} \mathrm{P}<0.01$. circ-ABCB10, circular RNA ABCB10; miR-1271, microRNA 1271; NC, negative control; OD, optical density; shRNA, short hairpin RNA; OE, overexpression; NS, not significant. 


\section{SKOV3 cells}

A

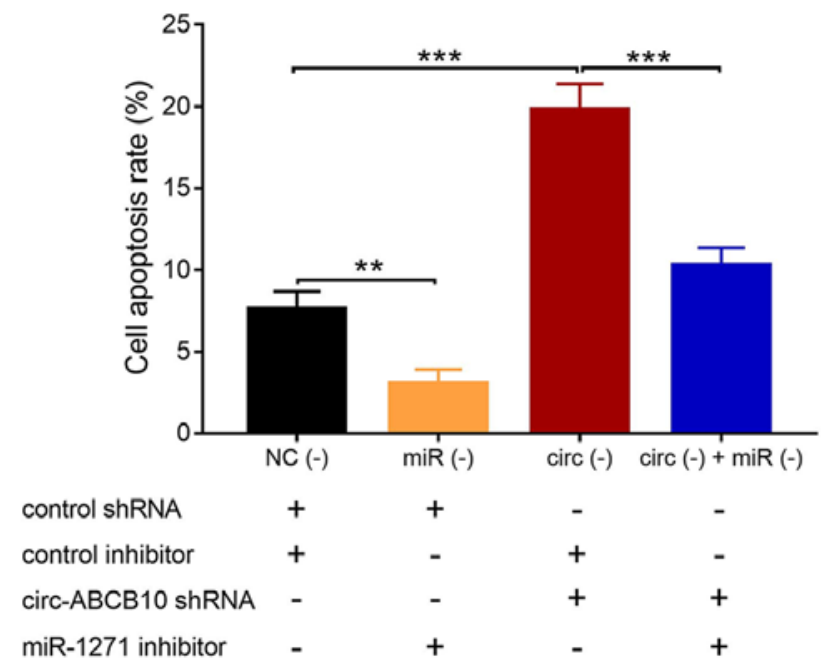

B

$\begin{array}{lll}\text { control shRNA } & + & + \\ \text { control inhibitor } & + & - \\ \text { circ-ABCB10 shRNA } & - & - \\ \text { miR-1271 inhibitor } & - & +\end{array}$

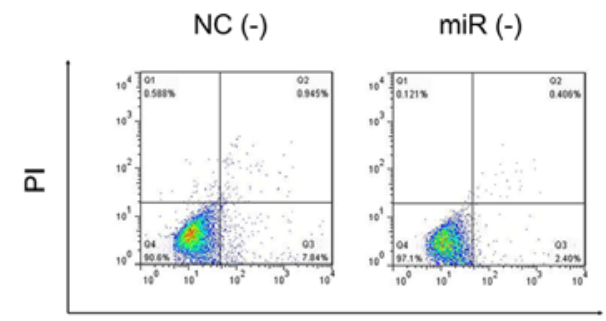

AV

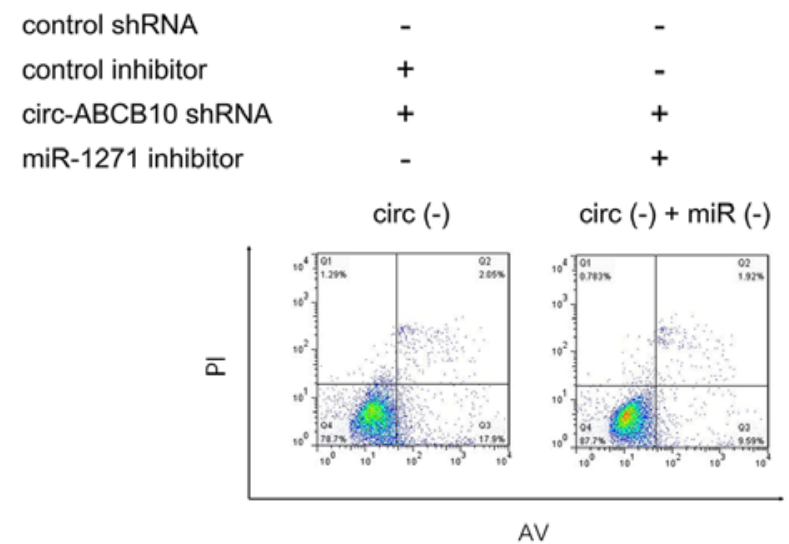

UWB1.289 cells

C

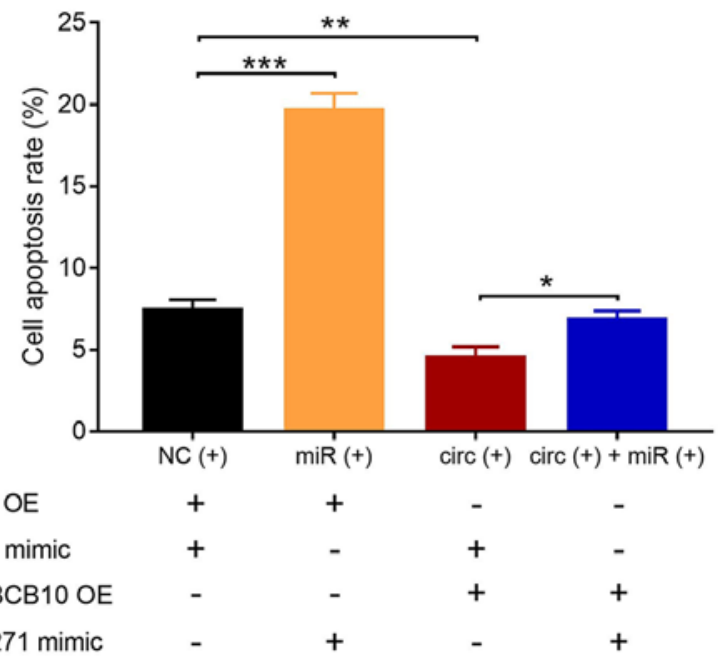

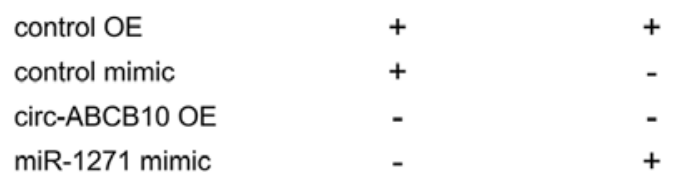

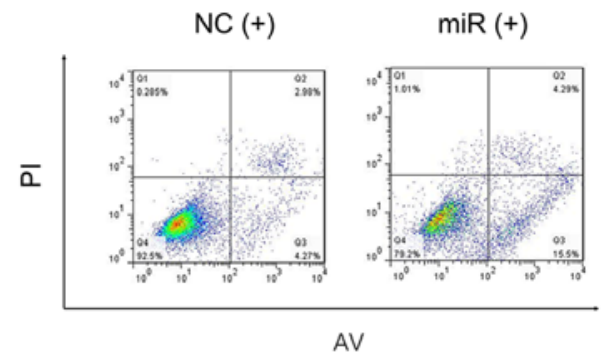

control OE

control mimic circ-ABCB10 OE miR-1271 mimic

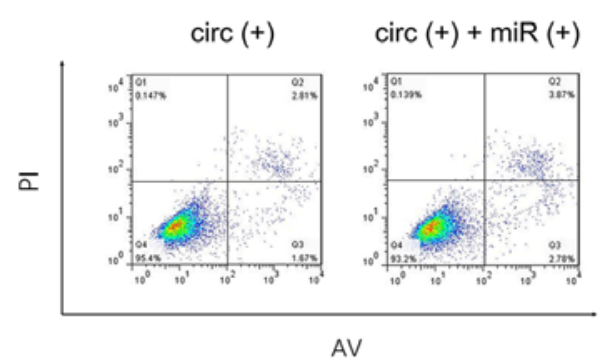

Figure 4. Effect of circ-ABCB10 and miR-1271 on cell apoptosis. (A) Cell apoptosis in NC (-), miR (-), circ (-) and circ (-) + miR (-) SKOV3 cells. (B) Representative flow cytometry plots in SKOV3 cells. (C) Cell apoptosis in NC (+), miR (+), circ (+) and circ (+) + miR (+) UWB1.289 cells. (D) Representative flow cytometry plots in UWB1.289 cells. ${ }^{*} \mathrm{P}<0.05,{ }^{* *} \mathrm{P}<0.01,{ }^{* * * *} \mathrm{P}<0.001$. circ-ABCB10, circular RNA ABCB10; miR-1271, microRNA 1271; NC, negative control; shRNA, short hairpin RNA; OE, overexpression; PI, propidium iodide; AV, Annexin V.

indicated that miR-1271 reversed the proproliferative effect of circ-ABCB10 in EOC.

circ-ABCB10 inhibits cell apoptosis by targeting miR-1271. In SKOV3 cells, cell apoptosis was significantly decreased in the miR (-) group compared with the NC (-) group, and in the circ (-) + miR (-) group compared with the circ (-) group. By contrast, cell apoptosis was significantly increased in the circ (-) group compared with the NC (-) group (Fig. 4A and B). In UWB1.289 cells, cell apoptosis was significantly increased 


\section{SKOV3 cells}

A

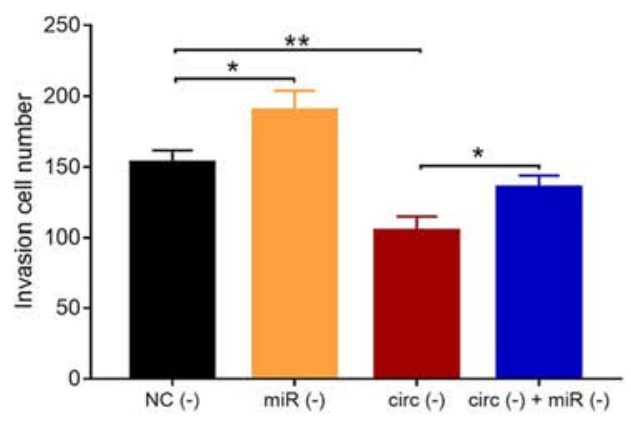

control shRNA

$+$

trol inhibitor

miR-1271 inhibitor

B

\begin{abstract}
control inhibitor circ-ABCB10 shRNA miR-1271 inhibitor
\end{abstract}

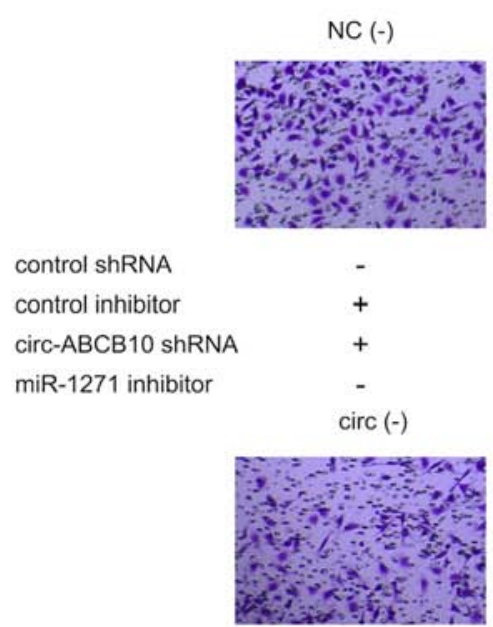

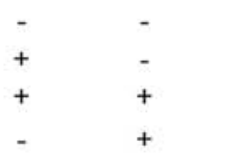

\section{UWB1.289 cells}

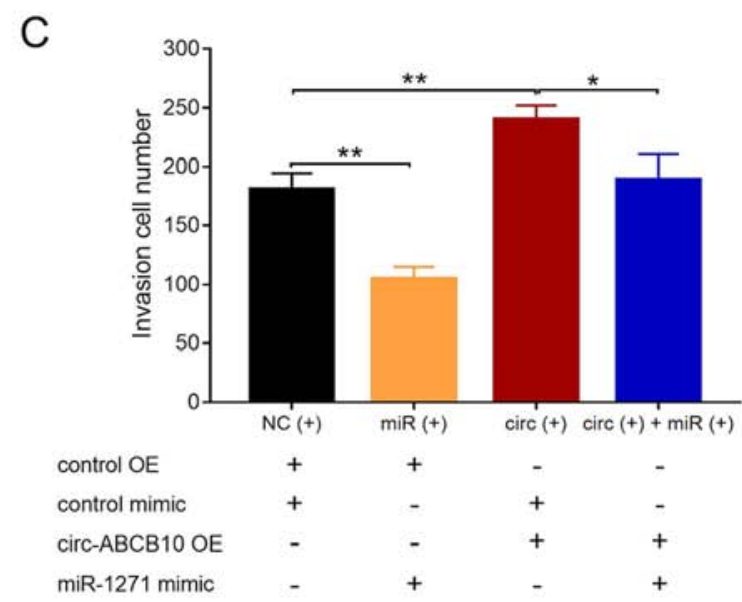

D control mimic
circ-ABCB10 OE
miR-1271 mimic $\operatorname{miR}(-)$
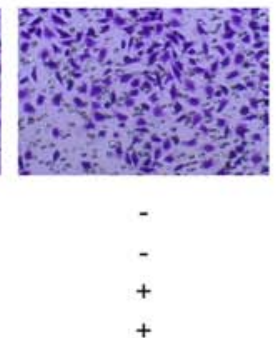

$\operatorname{circ}(-)+\operatorname{miR}(-)$

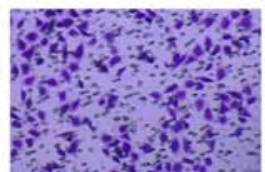

Figure 5. Effect of circ-ABCB10 and miR-1271 on cell invasion. (A) Cell invasion in NC (-), miR (-), circ (-) and circ (-) + miR (-) SKOV3 cells. (B) Representative images of the Transwell assay in SKOV3 cells under x200 magnification. (C) Cell invasion in NC (+), miR (+), circ (+) and circ (+) + miR (+) UWB1.289 cells. (D) Representative images of the Transwell assay in UBW1.289 cells under $\mathrm{x} 200$ magnification. ${ }^{~} \mathrm{P}<0.05,{ }^{* * *} \mathrm{P}<0.01$. circ-ABCB10, circular RNA ABCB10; miR-1271, microRNA 1271; NC, negative control; shRNA, short hairpin RNA; OE, overexpression.

in the miR $(+)$ group compared with the $\mathrm{NC}(+)$ group, and in the circ $(+)+$ miR $(+)$ group compared with the circ $(+)$ group. Moreover, cell apoptosis was significantly reduced in the circ (+) group compared with the NC (+) group (Fig. 4C and D). Collectively, the results suggested that miR-1271 attenuated the antiapoptotic effect of circ-ABCB10 in EOC.

circ-ABCB10 promotes cell invasion by targeting miR-1271. In SKOV3 cells, cell invasion was significantly increased in the miR (-) group compared with the NC (-) group, and in the circ (-) + miR (-) group compared with the circ (-) group. By contrast, cell invasion was significantly decreased in the circ (-) group compared with the NC (-) group (Fig. 5A and B). In UWB1.289 cells, cell invasion was significantly decreased in the miR (+) group compared with the NC (+) group, and in the circ $(+)+$ miR $(+)$ group compared with the circ $(+)$ group, but significantly increased in the circ $(+)$ group compared with the NC (+) group (Fig. 5C and D). Collectively, the results indicated that miR-1271 reversed the proinvasive effect of circ-ABCB10 in EOC.

circ-ABCB10 promotes the Capn $4 /$ Wnt/ $\beta$-catenin signaling pathway by targeting miR-1271. In SKOV3 cells, Capn4, Wnt and $\beta$-catenin expression levels were significantly increased in the miR (-) group compared with the NC (-) group, and in the circ (-) + miR (-) group compared with the circ (-) group, but significantly decreased in the circ (-) group compared with the NC (-) group (Figs. 6A-D and S1). In UWB1.289 cells, Capn 4, Wnt and $\beta$-catenin expression levels were significantly decreased in the miR (+) group compared with the $\mathrm{NC}(+)$ group, and in the circ $(+)+$ miR (+) group compared with the circ $(+)$ group. By contrast, Capn4, Wnt and $\beta$-catenin expression levels were significantly increased in the circ (+) group compared with the $\mathrm{NC}(+)$ group 


\section{SKOV3 cells}

A

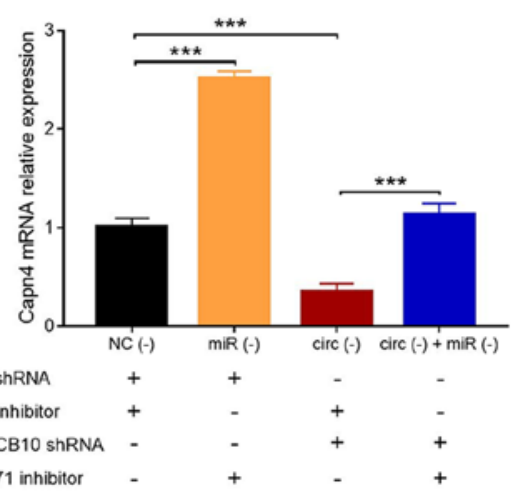

B

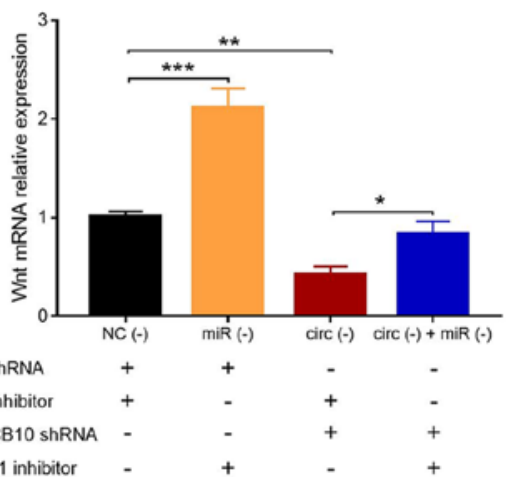

C

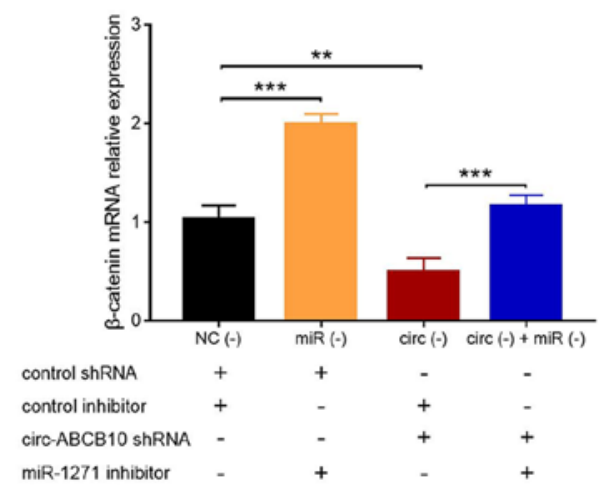

D

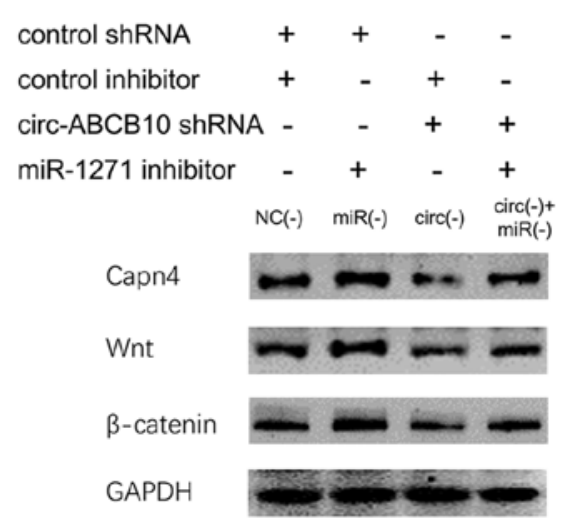

\section{UWB1.289 cells}

E

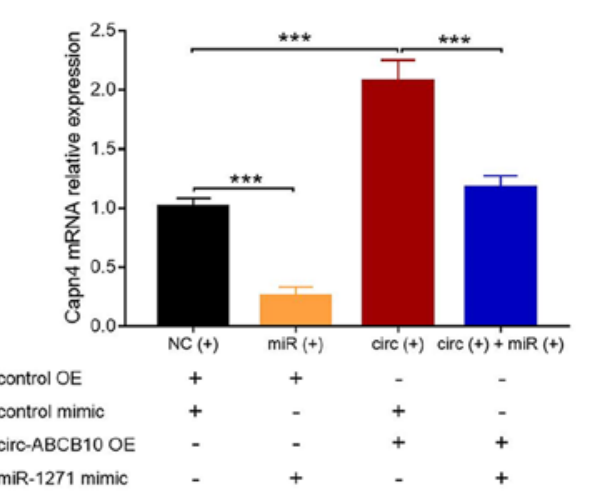

F

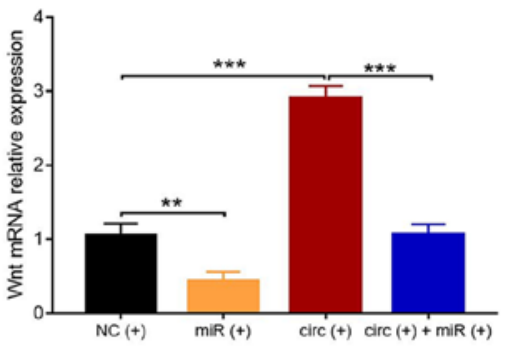

control OE

control mimic

circ-ABCB10 OE

miR-1271 mimic

G

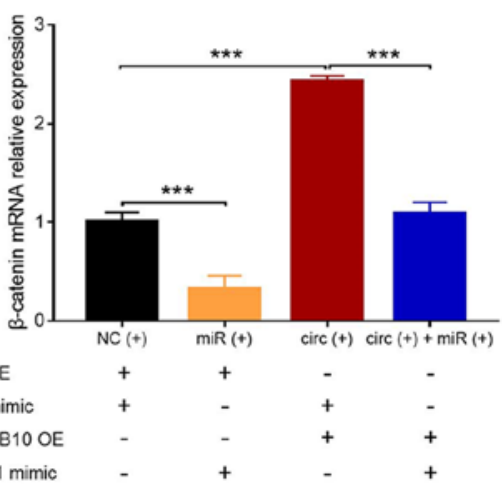

$\mathrm{H}$

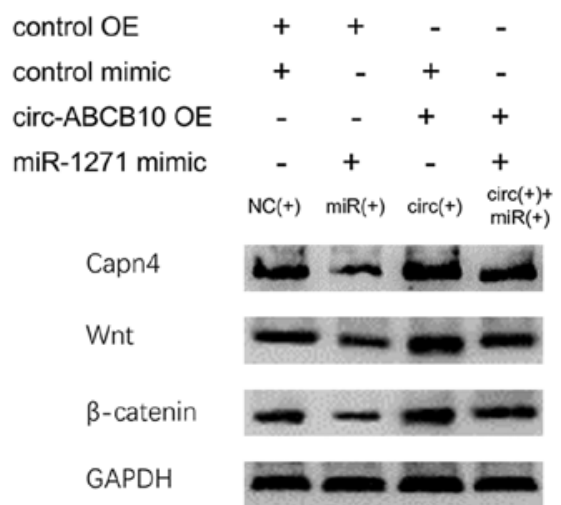

Figure 6. Effect of circ-ABCB10 and miR-1271 on the Capn4/Wnt/ $\beta$-catenin signaling pathway. mRNA expression levels of (A) Capn4, (B) Wnt and (C) $\beta$-catenin in SKOV3 cells. (D) Protein expression levels of Capn4, Wnt and $\beta$-catenin in SKOV3 cells. mRNA expression levels of (E) Capn4, (F) Wnt and $(G) \beta$-catenin in UBW1.289 cells. (H) Protein expression levels of Capn4, Wnt and $\beta$-catenin in UBW1.289 cells. ${ }^{*} \mathrm{P}<0.05$, ${ }^{* *} \mathrm{P}<0.01,{ }^{* * *} \mathrm{P}<0.001$. circ-ABCB10, circular RNA ABCB10; miR-1271, microRNA 1271; Capn4, calpain small subunit 1; NC, negative control; shRNA, short hairpin RNA; OE, overexpression. 

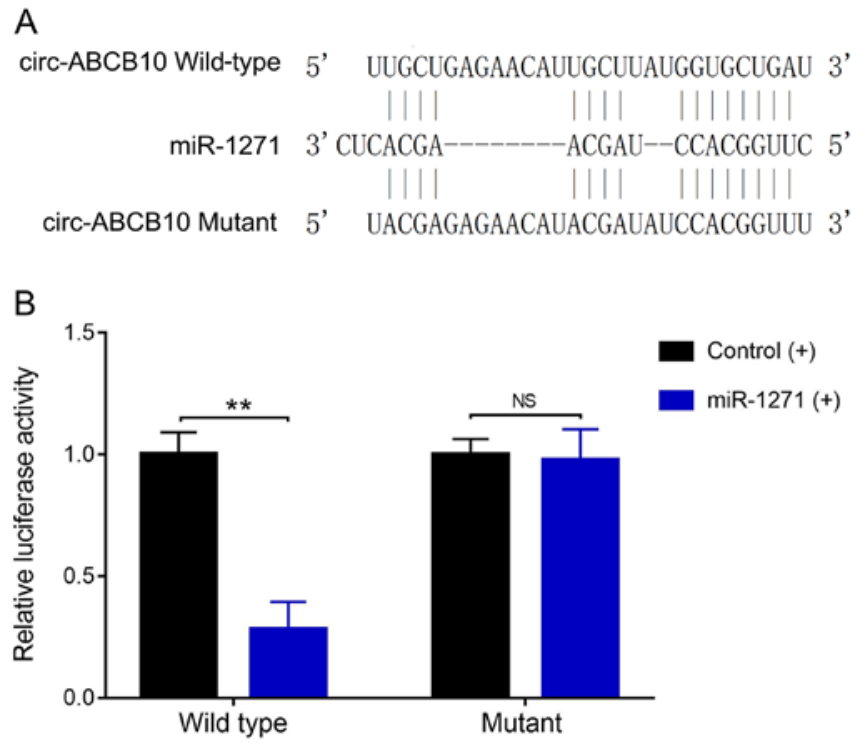

Figure 7. Interaction between circ-ABCB10 and miR-1271. (A) Sequence alignment of circ-ABCB10 and miR-1271. (B) Relative luciferase activity of wild-type/miR-1271 (+), wild-type/control (+), mutant/miR-1271 (+) and mutant/control (+) cells (B). ${ }^{* *} \mathrm{P}<0.01$. circ-ABCB10, circular RNA ABCB10; miR-1271, microRNA 1271; NS, not significant.

(Figs. 6E-H and S1). Collectively, the results suggested that miR-1271 reversed the positive regulation of circ-ABCB10 on the Capn4/Wnt/ $\beta$-catenin signaling pathway in EOC.

Direct interaction between circ-ABCB10 and miR-1271. To explore the interaction between circ-ABCB10 and miR-1271, miR-1271 and circ-ABCB10 binding sequences were designed (Fig. 7A). Subsequently, luciferase activity was evaluated, which revealed that luciferase activity was significantly reduced in wild-type/miR-1271 (+) cells compared with wild-type/control (+) cells. However, luciferase activity was not significantly different between mutant/miR-1271 (+) cells and mutant/control (+) cells (Fig. 7B). The results indicated that circ-ABCB10 directly bound to miR-1271 in EOC.

\section{Discussion}

EOC is a highly heterogeneous malignancy that is characterized by different precursor lesions, tissues of origin, molecular properties and clinical outcomes $(2,4)$. Despite advances in molecular predictors (for example, cancer antigen 125 and human epididymal protein 4), surgical debulking and chemotherapy for EOC in the past three decades, the clinical outcomes remain undesirable with increased chemoresistance and recurrence in patients with EOC $(2,13)$. To develop a novel and effective treatment strategy, the molecular pathogenesis underlying EOC requires further investigation.

With the application of high-throughput technologies, the underlying mechanisms and applications of circRNAs as therapeutic targets are widely explored in various diseases (14). circRNA, a covalently closed non-coding RNA, is generated by non-random back-splice events, is resistant to RNA degradation and has limited protein coding ability (15). circRNAs either function as oncogenic stimuli or tumor suppressors in cancer development and progression by sponging miRNAs to regulate the activities of downstream target genes or protein expression $(6,15)$. circ-ABCB10 is involved in the tumorigenesis of various types of cancer (6-8). In breast cancer, circ-ABCB10 knockdown inhibits breast cancer cell proliferation but facilitates apoptosis by interacting with miR-1271 (7). In non-small cell lung cancer, circ-ABCB10 expression is upregulated in NSCLC cell lines, which enhances cell proliferation and migration via sponging miR-1252 to upregulate forkhead box 2 (FOXR2) synthase expression (8). As for EOC, our previous study uncovered that circ-ABCB10 expression is elevated in EOC cell lines compared with normal ovarian epithelial cells, and it increases cell proliferation, but decreases apoptosis and the expression of miR-1271, miR-1252 and miR-203 (9). miR-1271 serves as a tumor suppressive miRNA, which inhibits cell proliferation and invasion via modulating downstream target genes in various types of cancer $(10,16,17)$. In EOC, miR-1271 inhibits cell viability, invasion and epithelial mesenchymal transition by directly binding to the 3'-untranslated region of zinc finger E-box binding homeobox $1 \mathrm{mRNA}$ in EOC (17). In colorectal cancer, miR-1271 promotes cell proliferation and invasion by suppressing Capn4 (10). Capn4 facilitates cell proliferation and migration in EOC by activating the Wnt/ $\beta$-catenin signaling pathway (11). Therefore, it was hypothesized that circ-ABCB10 might promote the progression of EOC via targeting miR-1271, Capn 4 and the Wnt/ $\beta$-catenin signaling pathway.

In the present study, the results indicated that circ-ABCB10 expression was upregulated, whereas miR-1271 expression was downregulated in EOC cells compared with normal ovarian cells. To investigate the role of the interaction between circ-ABCB10 and miR-1271 in cell proliferation, invasion and apoptosis, rescue experiments were performed. circ-ABCB10 negatively regulated miR-1271, whereas miR-1271 did not regulate circ-ABCB10 in EOC. Furthermore, circ-ABCB10 enhanced cell proliferation and invasion, but inhibited cell apoptosis in EOC. By contrast, miR-1271 suppressed cell proliferation and invasion, and facilitated cell apoptosis in EOC. miR-1271 reversed the proproliferative, proinvasive and antiapoptotic effects of circ-ABCB10 in EOC. Collectively, the results suggested that circ-ABCB10 facilitated cell proliferation and invasion, but suppressed cell apoptosis by negatively regulating miR-1271 in EOC. A few potential explanations for the results of the present study include: i) circ-ABCB10 might affect the function of its host gene ABCB10, which facilitates EOC tumor progression; and ii) circ-ABCB10 inhibited miR-1271 expression, which downregulated its downstream proteins (e.g. Capn4, FOXR2, regulator of G-protein signaling 17 and spindlin 1), resulting in enhanced tumor growth, metastasis and invasion of EOC $(8,10,18,19)$. The results also indicated that circ-ABCB10 increased Capn4 expression by repressing miR-1271.

Additionally, this study suggested that circ-ABCB10 promoted the Capn $4 / \mathrm{Wnt} / \beta$-catenin signaling pathway, whereas miR-1271 repressed the Capn4/Wnt/ $\beta$-catenin signaling pathway. Moreover, miR-1271 reversed the positive regulation of circ-ABCB10 on the Capn4/Wnt/ $\beta$-catenin signaling pathway in EOC. Collectively, the results indicated that circ-ABCB10 facilitated the Capn4/Wnt/ $\beta$-catenin signaling pathway by downregulating miR-1271 in EOC. To further 
confirm the sponging effect of circ-ABCB10 on miR-1271, a luciferase activity assay was performed, which indicated that circ-ABCB10 directly bound to miR-1271 in EOC. Herein, several explanations were proposed: i) circ-ABCB10 upregulated the Capn $4 / \mathrm{Wnt} / \beta$-catenin signaling pathway and subsequently induced mutations of the $\beta$-catenin gene (CTNNB1), which promoted tumor cell proliferation, migration and invasion via matrix metalloproteinase-2 (20); and ii) circ-ABCB10 promoted $\mathrm{Wnt} / \beta$-catenin pathway signaling and amplified the activation of target genes (such as c-Myc and cyclin-D1), which facilitated the malignant progression of EOC (21).

In conclusion, this study indicated that circ-ABCB10 promoted cell proliferation and invasion, but suppressed apoptosis by regulating the miR-1271-mediated Capn $4 / \mathrm{Wnt} / \beta$-catenin signaling pathway in EOC, highlighting the involvement of the circ-ABCB10/miR-1271/Capn4/Wnt/ $\beta$-catenin signaling pathway in the progression of EOC.

\section{Acknowledgements}

Not applicable.

\section{Funding}

No funding was received.

\section{Availability of data and materials}

The datasets used and/or analyzed during the current study are available from the corresponding author on reasonable request.

\section{Authors' contributions}

XX conceived and designed the present study. XL and YC performed the experiments and analyzed the data. XY interpreted the data and wrote the manuscript. All authors read and approved the final manuscript.

\section{Ethics approval and consent to participate}

Not applicable.

\section{Patient consent for publication}

Not applicable.

\section{Competing interests}

The authors declare that they have no competing interests.

\section{References}

1. Gloss BS and Samimi G: Epigenetic biomarkers in epithelial ovarian cancer. Cancer Lett 342: 257-263, 2014.

2. Krzystyniak J, Ceppi L, Dizon DS and Birrer MJ: Epithelial ovarian cancer: The molecular genetics of epithelial ovarian cancer. Ann Oncol 27 (Suppl 1): i4-i10, 2016.
3. Epidemiology Working Group Steering Committee, Ovarian Cancer Association Consortium Members of the EWG SC, Doherty JA, Jensen A, Kelemen LE, Pearce CL, Poole E, Schildkraut JM, Terry KL, Tworoger SS, et al: Current gaps in ovarian cancer epidemiology: The need for new population-based research. J Natl Cancer Inst 109: djx144, 2017.

4. Lheureux S, Gourley C, Vergote I and Oza AM: Epithelial ovarian cancer. Lancet 393: 1240-1253, 2019.

5. Khairallah AS, Genestie C, Auguste A and Leary A: Impact of neoadjuvant chemotherapy on the immune microenvironment in advanced epithelial ovarian cancer: Prognostic and therapeutic implications. Int J Cancer 143: 8-15, 2018.

6. Huang Y,Zhang Y, Jia L, Liu C and Xu F: Circular RNA ABCB10 promotes tumor progression and correlates with pejorative prognosis in clear cell renal cell carcinoma. Int J Biol Markers 34: 176-183, 2019.

7. Liang HF, Zhang XZ, Liu BG, Jia GT and Li WL: Circular RNA circ-ABCB10 promotes breast cancer proliferation and progression through sponging miR-1271. Am J Cancer Res 7: 1566-1576, 2017.

8. Tian X, Zhang L, Jiao Y, Chen J, Shan Y and Yang W: CircABCB10 promotes nonsmall cell lung cancer cell proliferation and migration by regulating the miR-1252/FOXR 2 axis. J Cell Biochem 120: 3765-3772, 2019.

9. Chen Y, Ye X, Xia X and Lin X: Circular RNA ABCB10 correlates with advanced clinicopathological features and unfavorable survival, and promotes cell proliferation while reduces cell apoptosis in epithelial ovarian cancer. Cancer Biomark 26: 151-161, 2019.

10. Li J, Xu J, Yan X, Jin K, Li W and Zhang R: Suppression of Capn4 by microRNA-1271 impedes the proliferation and invasion of colorectal cancer cells. Biomed Pharmacother 99: 162-168, 2018.

11. Yang X, Sun J, Xia D, Can X, Liu L, Zhang J, Xu H, Du N, Liu W, Shen F, et al: Capn4 enhances osteopontin expression through activation of the $\mathrm{Wnt} / \beta$-catenin pathway to promote epithelial ovarian carcinoma metastasis. Cell Physiol Biochem 42: 185-197, 2017.

12. Livak KJ and Schmittgen TD: Analysis of relative gene expression data using real-time quantitative PCR and the 2(-Delta Delta C(T)) method. Methods 25: 402-408, 2001.

13. Dochez V, Caillon H, Vaucel E, Dimet J, Winer $\mathrm{N}$ and Ducarme G: Biomarkers and algorithms for diagnosis of ovarian cancer: CA125, HE4, RMI and ROMA, a review. J Ovarian Res 12: 28, 2019

14. Zhao ZJ and Shen J: Circular RNA participates in the carcinogenesis and the malignant behavior of cancer. RNA Biol 14: 514-521, 2017.

15. Chen B and Huang S: Circular RNA: An emerging non-coding RNA as a regulator and biomarker in cancer. Cancer Lett 418: 41-50, 2018.

16. Liu X, Ma L, Rao Q, Mao Y, Xin Y, Xu H, Li C and Wang X: miR-1271 inhibits ovarian cancer growth by targeting cyclin G1. Med Sci Monit 21: 3152-3158, 2015.

17. Jiao Y,Zhu G,YuJ,Mao Y,Xin Y,XuH,LiCandWang X:miR-1271 inhibits growth, invasion and epithelial-mesenchymal transition by targeting ZEB1 in ovarian cancer cells. Onco Targets Ther 12: 6973-6980, 2019

18. Chi Y, Jin Q, Liu X, Xu L, He X, Shen Y, Zhou Q, Zhang J and Jin M: miR-203 inhibits cell proliferation, invasion, and migration of non-small-cell lung cancer by downregulating RGS17. Cancer Sci 108: 2366-2372, 2017.

19. Du HY and Liu B: miR-1271 as a tumor suppressor in breast cancer proliferation and progression via targeting SPIN1. Eur Rev Med Pharmacol Sci 22: 2697-2706, 2018.

20. Bodnar L, Stanczak A, Cierniak S, Smoter M, Cichowicz M, Kozlowski W, Szczylik C, Wieczorek M and Lamparska-Przybysz M: Wnt/3-catenin pathway as a potential prognostic and predictive marker in patients with advanced ovarian cancer. J Ovarian Res 7: 16, 2014.

21. Arend RC,Londoño-Joshi AI, Straughn JM Jr and Buchsbaum DJ: The Wnt/ $\beta$-catenin pathway in ovarian cancer: A review. Gynecol Oncol 131: 772-779, 2013.

This work is licensed under a Creative Commons Attribution-NonCommercial-NoDerivatives 4.0 International (CC BY-NC-ND 4.0) License. 